\title{
miRNA-7062-5p Promoting Bone Resorption After Bone Metastasis of Colorectal Cancer Through Inhibiting GPR65
}

OPEN ACCESS

Edited by:

Na Luo,

Nankai University, China

Reviewed by:

Huai-Qiang Ju,

Sun Yat-sen University, China

Xiawei Wei,

Sichuan University, China

*Correspondence:

Lili Zhang

lili_home20@163.com

Ziming Wang

zm_wang72@163.com

Specialty section:

This article was submitted to

Molecular Medicine,

a section of the journal

Frontiers in Cell and Developmental

Biology

Received: 17 March 2021

Accepted: 06 May 2021

Published: 17 August 2021

Citation:

Chen L, Wang Y, Lu X, Zhang L and Wang $Z$ (2021) miRNA-7062-5p

Promoting Bone Resorption After Bone Metastasis of Colorectal Cancer

Through Inhibiting GPR65.

Front. Cell Dev. Biol. 9:681968.

doi: 10.3389/fcell.2021.681968

\author{
Liang Chen ${ }^{1}$, Yu Wang ${ }^{1}$, Xingchen Lu' ${ }^{1}$, Lili Zhang ${ }^{2 *}$ and Ziming Wang ${ }^{1 *}$ \\ 'Department of Orthopedics, Army Medical Center, Army Medical University, Chongqing, China, ${ }^{2}$ Department of Military \\ Psychology, College of Psychology, Army Medical University, Chongqing, China
}

Bone metastasis is positively associated with a poor prognosis in patients with colorectal cancer (CRC). CRC always leads to osteolytic change, which is regulated by aberrant activation of osteoclasts. MicroRNAs are remarkedly involved in metastasis of CRC; however, their role in bone metastasis of CRC is still unclear. The aim of this study is to find key microRNAs that are critical to bone resorption in bone metastasis of CRC. In this study, bone metastasis model was established through intratibially injecting CT-26 cells or MC-38 cells. Tartrate-resistant acid phosphatase (TRAP) staining was performed to explore the osteoclastogenesis of primary early osteoclast precursors (OCPs) after stimulation by CT-26 conditioned medium (CM). Then, microarray assay was performed to find differentially expressed miRNAs and mRNAs. The target gene of miRNA was confirmed by dual-luciferase analysis. The effect of miRNA, its target gene on osteoclastogenesis, and involved pathways were explored by Western blot, immunofluorescence analysis, and TRAP staining. Finally, the effect of miRNA on bone resorption in vivo was observed. miRNA-7062-5p was upregulated in early OCPs cultured in CT-26 CM or MC-38 CM. GPR65 was proven to be the target gene of miRNA-7062-5p. Overexpression of GPR65 can rescue the osteoclastogenesis caused by miRNA-7062-5p through activation of AMPK pathway. Local injection of miRNA7062-5p inhibitors efficiently improved the bone resorption. Our study found the role of miRNA-7062-5p in regulating osteoclast formation, and our findings provided a potential therapeutic target in treatment of bone metastasis of CRC.

Keywords: miRNA-7062-5p, GPR65, colorectal cancer, bone metastasis, osteoclast

\section{INTRODUCTION}

Although the incidence of bone metastasis from colorectal cancer (CRC) is relatively rare in clinic, the prognosis is pessimistic. Patients with CRC presenting with bone metastasis are often younger and have a poor prognosis; 5-year survival rate was only 5.7\% (Kawamura et al., 2018). Moreover, the treatment is insufficient, partly because the underlying mechanism of bone metastasis of CRC is still clearly unexplored.

Abbreviations: CRC, colorectal cancer; TRAP, tartrate-resistant acid phosphatase; CM, conditioned medium; OCPs, osteoclast precursors; M-CSF, macrophage colony stimulating factor; RANKL, receptor activator of nuclear factor- $\mathrm{B}$ ligand; MMP, matrix metalloproteinase. 
In bone microenvironment, the balance between osteoblasts and osteoclasts maintains the homeostasis of bone remodeling. CRC is a kind of osteolytic tumors. For these types of tumors, aberrant activation of osteoclasts promotes the bone resorption and pathologic fractures (Clohisy et al., 1996; Li et al., 2019b). Osteoclasts derive from monocytes/macrophage lineage. RANKL determines monocytes/macrophages to commit to osteoclast precursors (OCPs) (Liang et al., 2019). In tumor microenvironment, cancer cells can directly promote the osteoclastogenesis of OCPs through RANKL-dependent or independent ways (Liang et al., 2019). However, little is known on how the osteoclasts or its precursors change in CRC microenvironment.

MicroRNAs remarkedly participate in the progression and metastasis of $\mathrm{CRC}$ and are important regulators for osteoclastogenesis. Some microRNAs, such as miRNA-802, miRNA-875-3p, miRNA-574, miRNA-708, miRNA-206, and miRNA-27b, can attenuate the progression of CRC, whereas miRNA-503 facilitates the tumor growth (Ye et al., 2013; Park et al., 2018; Li et al., 2019a, 2020b; Sun et al., 2019; Zhang et al., 2020). In addition, miRNAs can also be used to predict the prognosis of CRC. It was also reported that miRNA-483, miRNA-218, miRNA-199a-5p, miRNA-133a, and miRNA340 can regulate the osteoclast formation (Zhao et al., 2017; Guo et al., 2018, 2019; Li et al., 2018, 2020a). Considering the importance of miRNAs in both tumor progression and osteoclastogenesis, it is worthy to know the function of miRNAs in bone metastasis of CRC.

In this study, we identified that miRNA-7062-5p was treated by condional medium collected from CRC and promoted the osteoclastogenesis through targeting GPR65. Downregulating miRNA-7062-5p efficiently attenuated the progression of bone destruction caused by CRC.

\section{MATERIALS AND METHODS}

\section{Animals}

All procedures involving mice and experimental protocols were approved by the Institutional Animal Care and Use Committee of Daping Hospital at Army Medical University. C57BL/6 or $\mathrm{BALB} / \mathrm{c}$ mice for all experiments were 6-8 weeks of age. CT-26 and MC-38 cells are two commonly used CRC cell lines. Thus, for bone metastasis studies, 500,000 CT-26 or MC-38 cells were resuspended in phosphate-buffered saline (PBS) and injected into tibias of mice after anesthetization. Antagomir-7062-5p or scrambled controls dissolved in PBS $(10 \mu \mathrm{g} / 50 \mu \mathrm{L})$ were injected intravenously every 3 days for continuous 3 weeks. At specific timepoints, mice were killed, and the hindlimbs were removed and fixed in $4 \%$ paraformaldehyde for histochemical staining or tartrate-resistant acid phosphatase (TRAP) staining. Five to 10 mice were used per group.

\section{Histological Analysis}

Tibias were dissected and fixed for $48 \mathrm{~h}$. Then, the samples were decalcified by daily change of $15 \%$ tetrasodium EDTA for 3 weeks and embedded in paraffin. For safranin $\mathrm{O}$ and fast green staining, samples were cleared twice in xylene and were rehydrated by passage through an ethanol series following by immersion in PBS. The samples were then stained in $0.1 \%$ safranin $\mathrm{O}$ solution for $3 \mathrm{~min}$ and washed in PBS three times. Next, the samples were immersed in $0.1 \%$ fast green solution for $10 \mathrm{~s}$ and separated in $1 \%$ acetum. The samples were then observed and captured. The TRAP staining was performed according to the manufacturers' instructions (BestBio Biotechnology, Beijing, China). For immunofluorescence analysis, early OCPs were seeded on coverslips and transfected by miRNAs. Then, the samples were fixed in $4 \%$ paraformaldehyde for $15 \mathrm{~min}$ in $4^{\circ} \mathrm{C}$. After paraformaldehyde was discarded, the samples were washed in PBS and incubated in blocking buffer containing $10 \%$ donkey serum and 5\% bovine serum albumin for $1 \mathrm{~h}$ in $37^{\circ} \mathrm{C}$. Then, primary antibody was incubated overnight in $4^{\circ} \mathrm{C}$. Secondary antibody was added after the samples were washed in PBS followed by staining with Hoechst 33342 $(1: 1,000)$ for $30 \mathrm{~min}$. Primary antibody used was anti-GPR65 at 1:200 (Biorbyt, Cambridgeshire, United Kingdom). Secondary antibody was donkey anti-rabbit conjugated with $\mathrm{Cy} 3$ at 1:200 (Jackson ImmunoResearch Laboratories, Inc., West Grove, PA, United States).

\section{Primary Early OCP Isolation and Culture}

Bone marrows were rinsed out from marrow cavity of tibias and femurs by sterilized PBS. The cell suspension was collected and centrifuged at $500 \mathrm{~g}$ for $5 \mathrm{~min}$. After discarding the supernatant, erythrocytes were removed. The cells were stained using CD115 antibody conjugated with APC and RANKL antibody conjugated with PE (Biolegend, San Diego, CA, United States) for $1 \mathrm{~h}$ in $4^{\circ} \mathrm{C}$. Then, the primary early OCPs labeled as CD115-positive and RANKL-negative were sorted out by FACS.

Isolated early OCPs were cultured in Dulbecco modified eagle medium (DMEM) supplemented with $10 \%$ fetal bovine serum, $100 \mathrm{U} / \mathrm{mL}$ penicillin, and $100 \mathrm{U} / \mathrm{mL}$ streptomycin (Gibco, Thermo Fisher Scientific, Waltham, MA, United States) and macrophage colony-stimulating factor (M-CSF) (50 ng/mL) (Biolegend, San Diego, CA, United States). For osteoclastogenic induction, early OCPs were stimulated by M-CSF $(50 \mathrm{ng} / \mathrm{mL})$ and RANKL (50 ng/mL) for at least 4-6 days. In some experiments, early OCPs under osteoclastogenic induction were cultured with conditioned medium (CM) from CT-26 for 4 days.

\section{Transient Transfection}

Agomir-7062-5p, antagomir-7062-5p and their scrambled controls, siRNA pool, and pcDNA3.1 (+) plasmid containing GPR65 were synthesized by GenePharma, Co. (Shanghai, China). Transfection was performed using Lipofectamine RNAiMax Reagent (Invitrogen, Thermo Fisher Scientific). The siRNA sequence for AMPK $\alpha$ : 5'-CUAUGAAUGGAAGGUUGUA-3', $5^{\prime}$-UACAACCUUCCAUUCAUA-3'.

\section{Luciferase Reporter Assay}

293T cells were seeded into 6-well plates. Cells were transfected with pGL3-GPR65 3' UTR and pRL-TK (Promega, Madison, WI, United States) Renilla luciferase plasmid, as well as wild-type (wt) or mutant (mut) mir-7062-5p or controls using Lipofectamine 
RNAiMax Reagent following the manufacturer's instructions. Luciferase assays were conducted using the dual-luciferase reporter assay system (Promega). The values of luminescent signals from firefly luciferase construct were normalized by Renilla luciferase assay.

\section{Quantitative Real-Time Polymerase Chain Reaction}

The total RNA was extracted from cells using TRIzol reagent (Invitrogen, Thermo Fisher Scientific). First-strand complementary DNA was reversed from $1 \mathrm{mg}$ of total RNA using PrimeScript RT reagent Kit (TakaraBio, Tokyo, Japan) according to the manufacturer's instructions. The stem-loop reverse transcriptase-polymerase chain reaction (RT-PCR) was used for quantification of miRNA-7062-5p. Quantitative real-time PCR was performed using an SYBR Green Premix Ex TaqTMII Kit (TakaraBio, Tokyo, Japan). GAPDH and U6 were used as normalization controls for mRNA and miRNA, respectively. The sequences of the primers are shown as follows: GAPDH (forward: 5'-TGGATTTGGACGCATTGGTC-3' and reverse: $5^{\prime}$-TTTGCACTGGTACGTGTTGAT-3'), cathepsin K (forward: 5'-CTGGCTGGGGTTATGTCTCAA-3', and reverse: 5'-GGCTACGTCCTTACACACGAG-3'), MMP-2 (forward: 5' TGACTTTCTTGGATCGGGTCG-3', and reverse: 5' ${ }^{\prime}$-AAGCA CCACATCAGATGACTG-3'), MMP-9 (forward: 5' CTATGGTTACACTCG-3' ${ }^{\prime}$, and reverse: 5'-GGCAGGGACAG TTGCTTCT-3').

\section{Western Blot}

The cells were lysed with RIPA buffer containing protease inhibitors (Beyotime, Shanghai, China). Total proteins were collected and subjected to sodium dodecyl sulfatepolyacrylamide gel electrophoresis and transferred to polyvinylidene difluoride membranes. The membranes were blocked and incubated with primary antibodies. After being washed, the membranes were then incubated with horseradish peroxidase-linked secondary antibodies. The antibodies used were as follows: GPR65 (1:1,000, Biorbyt), JNK (1:2,000; Santa Cruz, Dallas, TX, United States), phosphorylation JNK (1:2,000; Santa Cruz), AMPK $\alpha$ (Abcam, Cambridge, MA, United States), phosphorylation $\mathrm{AMPK} \alpha(), \mathrm{GAPDH}$ (Abcam), goat anti-rabbit immunoglobulin G (1:5,000; Abcam).

\section{Microarray Assay}

Total RNA was extracted from early OCPs. MiRNAs were isolated and labeled using biotin and detected with Ambion WT Expression Kit. MiRNA and mRNA microarray assays were completed by CNKINGBIO (Beijing, China). The miRNA 4.0 and Clariom D were used for microarray assay. The data sources of non-coding RNA were from Luo et al. (2013). The signal intensity of each spot was calculated by the program $\mathrm{R}$ (2.12.1). Spots that passed the criteria were normalized by the invariant set normalization method. Normalized spot intensities were transformed to gene expression $\log 2$ ratios between the control group and treatment group using the pairwise $t$-test.
The spots with a $\mid \log 2$ ratio $\mid \geq 0.585$ and a $p<0.05$ were selected for analysis.

\section{Statistical Analysis}

All data are representative of at least three experiments of similar results performed in triplicate unless otherwise indicated. Data are expressed as mean \pm SD. One-way analysis of variance was used to determine the significance of difference between results, with $* p<0.05$ being regarded as significant.

\section{RESULTS}

\section{Secreta Derived From CRC Cells Induce Osteoclastogenesis}

Few studies focused on the bone metastasis of CRC and the onset of osteolysis. For establishing bone metastasis model in mice, we intratibially injected Balb/c mice with CT-26 CRC cell line. First, early OCPs labeled with CD115-positive and RANKnegative were isolated through FACS (so-called early OCPs). Approximately $4.5 \%$ of early OCPs can be sorted out from bone marrow in normal mice (Figure 1A). As expected, during osteoclastogenic induction treated with RANKL and M-CSF for 6 days, early OCPs formed TRAP-positive multinuclear giant cells. Notably, TRAP-staining results suggested higher TRAP activity and more osteoclast number were identified in OCPs after indirect coculture with CT-26 cells (Figures 1B,C). In addition, relative TRAP activity assay confirmed that TRAP activity increased after indirectly coculturing with CT-26 cells (Figure 1D). Consistently, the mRNA levels of osteoclastogenic markers, MMP-2, MMP-9, and cathepsin K, were significantly upregulated in OCPs stimulated with CT-26 CM (Figures 1EG). These data suggested secreta from CRC cells induce osteoclastogenesis of OCPs.

\section{miRNA-7062-5p Promotes Osteoclastogenesis of Early OCPs}

To assess microRNAs involved in CRC cell-induced osteoclastogenesis, OCPs stimulated with CT-26 CM plus RANKL and M-CSF were collected. Microarray assays revealed that 60 miRNAs were differently expressed during differentiation. Among them, miRNA-7062-5p was the most obviously increased miRNA in CT-26 CM-treated OCPs (Figure 2A). To explore whether miRNA-7062-5p participates in CRC-induced osteoclastogenesis, CM was collected from two CRC cell lines, CT-26 and MC-38 cells, respectively. RT-PCR demonstrated the expression of miRNA-7062-5p upregulated in OCPs after stimulation with both CT-26 CM and MC-38 CM (Figure 2B). Notably, the level of miRNA-7062-5p in primary OCPs increased over time after injection of CT-26 or MC-38 cells intratibially (Figure 2C). These data indicated miRNA-7062-5p positively correlated with osteoclastogenesis and can be induced by secreta derived from CRC cells. To further assess whether miRNA-7062-5p plays a role in regulating osteoclastogenesis in tumor microenvironment, primary OCPs were treated with agomir-7062-5p or antagomir-7062-5p and then stimulated 


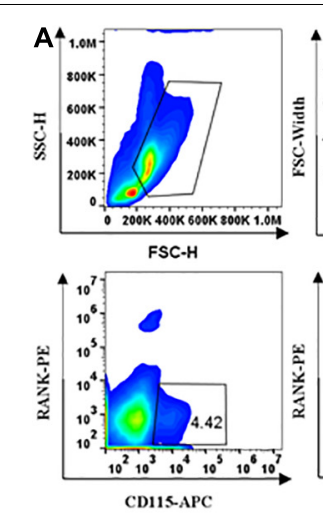

D

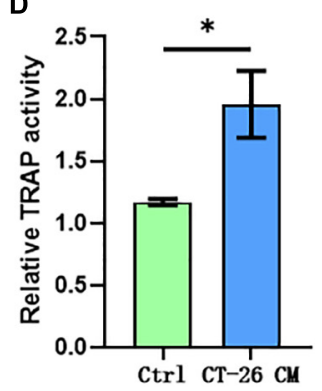

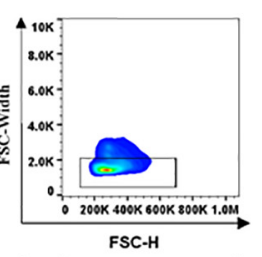

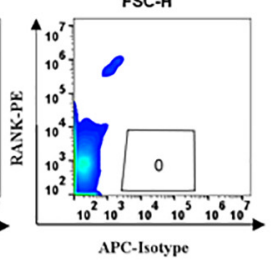

E

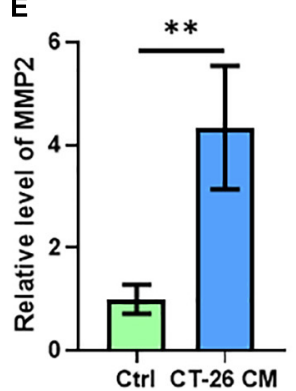

B

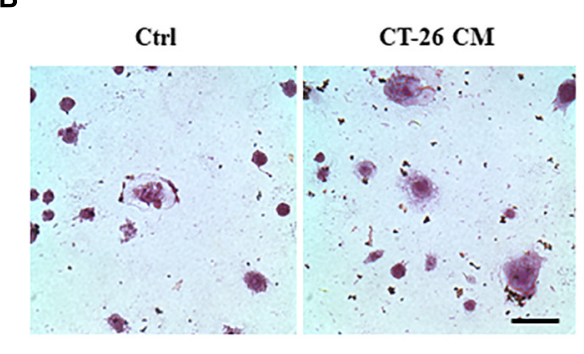

$F$

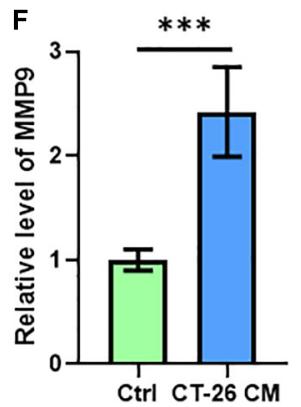

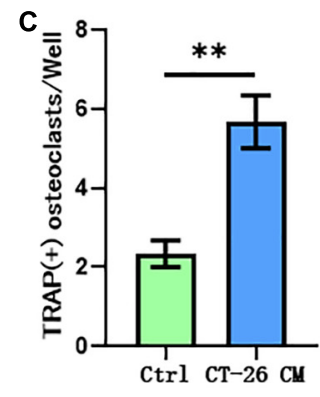

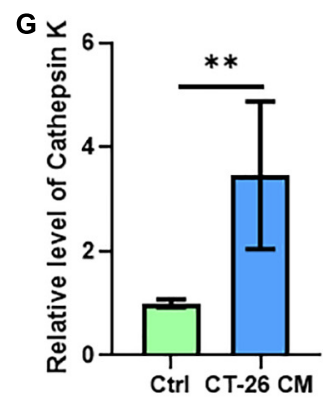

FIGURE 1 | Conditioned medium collected from CRC cell line, CT-26, promotes early osteoclast precursors to differentiate into osteoclasts. (A) Gating strategy for sorting out early osteoclast precursors. (B) Representative images of TRAP staining demonstrating osteoclast formation of early OCPs under stimulation of CT-26 CM or DMEM (scale bar $=250 \mu \mathrm{m}$ ). (C) Quantification of the number of TRAP-positive osteoclasts per well in (B). (D) Quantification of relative TRAP activity per well in (B). (E-G) The relative expression of osteoclastogenic biomarkers, MMP2, MMP9, and cathepsin $\mathrm{K}$, was determined by real-time PCR. * $p<0.05$, $* * p<0.01$, $* * * p<0.001$.

in CT-26 CM, as well as RANKL and M-CSF. TRAP staining and relative TRAP activity results indicated the number of osteoclasts, and TRAP activity significantly increased in the agomir-7062-5p-treated group and decreased in the antagomir7062-5p-treated group (Figures 2D-F), demonstrating that miRNA-7062-5p directly promoted osteoclastogenesis of OCPs.

\section{MiRNA-7062-5p Directly Targets GPR65}

To understand the underlying mechanism in miRNA-7062$5 \mathrm{p}$-activated osteoclastogenesis, transcriptomic profiling was analyzed in OCPs treated with CT-26 CM. We detected the differentially expressed genes related to osteoclastogenesis. Interestingly, our results showed the mRNA level of GPR65 downregulated approximately 25 folders (Figure 3A). As it was reported GPR65 negatively regulated osteoclastic activity in osteoporosis (Hikiji et al., 2014), it implied that GPR65 may play an important role in CRC-induced osteoclastogenesis. We next explored whether miRNA-7062-5p can target GPR65. Notably, online analysis tool (TargetScan ${ }^{1}$ ) predicted that GPR65 could be one potential target gene of miRNA-7062-5p (Figure 3B). Notably, the luciferase activity of $293 \mathrm{~T}$ cells that were transfected wt 3' UTR of GPR65 was remarkedly reduced by miRNA-7062$5 \mathrm{p}$, whereas the luciferase activity in group transfection with the mutated-type (mut) 3' UTR of GPR65 was not affected by miRNA-7062-5p (Figure 3C). In addition, up-regulation of

${ }^{1} \mathrm{http}: / /$ www.targetscan.org
miRNA-7062-5p significantly reduced the protein expression of GPR65 in OCPs; the immunofluorescence signals remarkedly reduced in the miRNA-7062-5p-treated group (Figures 3D,E). Together, these data indicated that GPR65 is the target gene of miRNA-7062-5p. Moreover, overexpression of miRNA-7062-5p can efficiently reduce the protein expression of GPR65 in OCPs.

\section{miRNA-7062-5p Regulates Osteoclastogenesis of OCPs Dependent on GPR65}

We demonstrated that GPR65 is the target gene of miRNA7062-5p, and then we performed rescue experiments to further assess that GPR65 is essential to miRNA-7062-5p-mediated osteoclastogenesis of OCPs. As expected, the protein level of GPR65 downregulated in OCPs after transfection with agomir7062-5p, whereas the expression of GPR65 was rescued after cotransfection with cDNA3.1(+) containing GPR65 in both normal and CT-26 CM-treated early OCPs (Figures 4A,B). Then, the differentiation of OCPs was investigated. TRAP staining showed that overexpression of GPR65 can decrease the increased number of osteoclasts that were promoted by miRNA-7062-5p transfection in the presence of CT-26 CM (Figures 4C,D). Meanwhile, relative TRAP activity supported the results in TRAP staining; the relative activity was enhanced in agomir-7062-5p-treated group and returned to the level in the control group after overexpression of GPR65 (Figure 4E). 

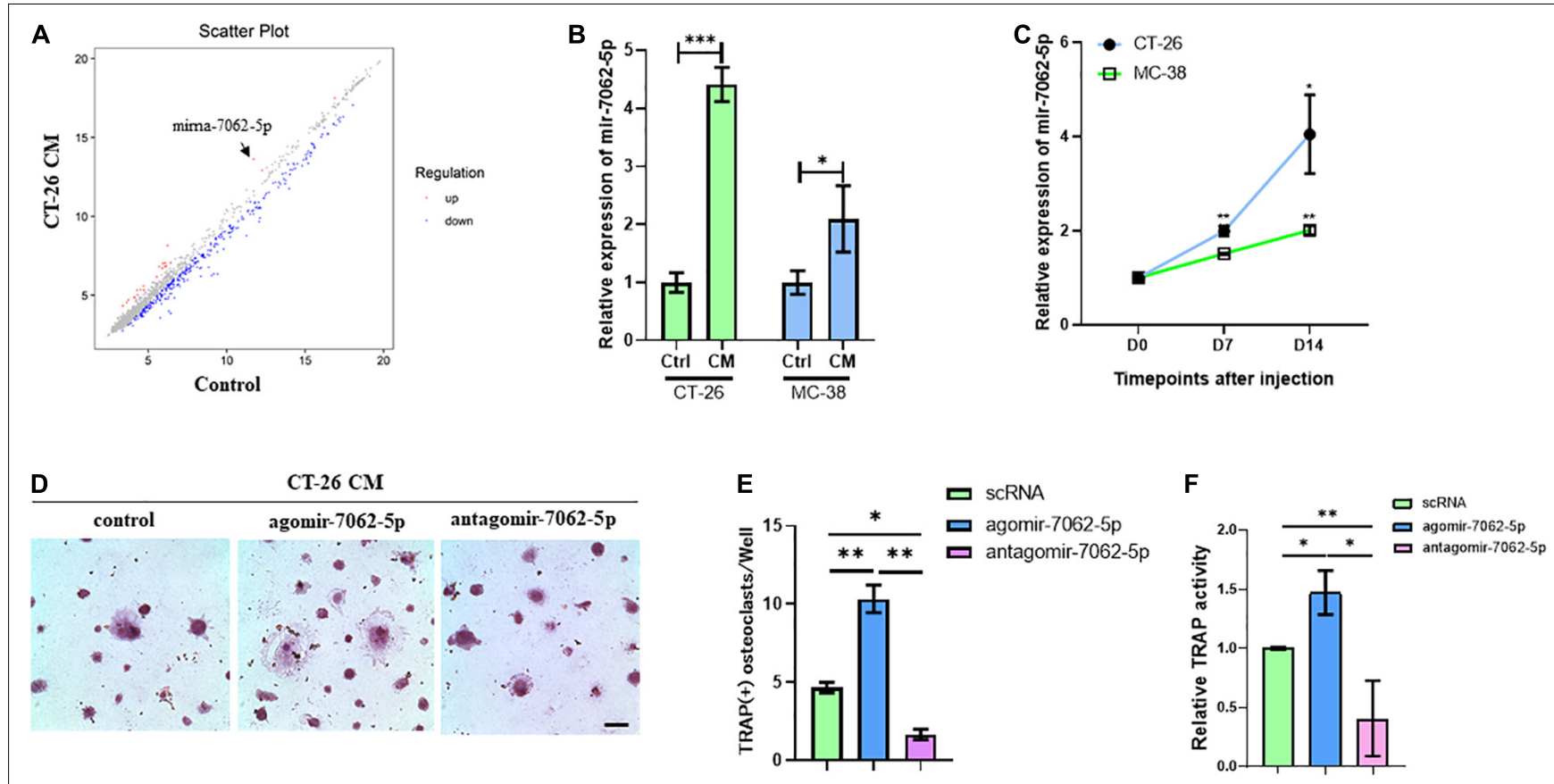

FIGURE 2 | The expression of miRNA-7062-5P upregulates during osteoclastogenesis induced by CT-26 CM and promotes osteoclast formation. (A) Scatter plots for microarray assay demonstrated differentially expressed miRNAs in early OCPs treated with CT-26 CM or DMEM. (B) The change folders of miRNA-7062-5p level in early OCPs after treatment by CT-26 CM or MC-38 CM compared with the control group. (C) The change of miRNA-7062-5p expression in sorted early OCPs at specific timepoints after injection of CT-26 cells or MC-38 cells into tibias. (D) Representative images of TRAP staining demonstrating osteoclastogenesis after early OCPs stimulated by RANKL and M-CSF in the presence of CT-26 CM after transfection with controls, agomir-7062-5p, or antagomir-7062-5p (scale bar $=250 \mu \mathrm{m}$ ). (E) Quantification of the number of TRAP positive osteoclasts per well in (D). (F) Quantification of relative TRAP activity per well in (D). ${ }^{*} p<0.05,{ }^{* *} p<0.01$, ${ }^{* * *} p<0.001$.

Then, we further proved that miRNA-7062-5p inhibition attenuated osteoclast formation, and this process was dependent on regulating GPR65. OCPs were treated with antagomir$7062-5 p$ or cotreated with siRNA targeting GPR65 in normal medium or CT-26 CM. Western blots showed the protein level of GPR65 in OCPs significantly upregulated after treatment with antagomir-7062-5p, whereas this upregulation can be prevented when cotransfected with siGPR65 (Figures 5A,B). TRAP staining showed miRNA-7062-5p inhibition can decrease the number of osteoclasts, whereas the number was rescued through inhibiting expression of GPR65 in the presence of CT-26 CM (Figures 5C,D). Furthermore, relative TRAP activity demonstrated that TRAP activity was downregulated in antagomir-7062-5p-treated group and recovered after cotransfection with siGPR65 (Figure 5E). These data proved that miRNA-7062-5p stimulated osteoclastogenesis of OCPs through targeting GPR65 in the presence of CT-26 CM.

\section{GPR65/AMPK $\alpha$ Signaling Inhibited Osteoclastogenesis of OCPs Through Preventing Activation of the JNK Pathway}

It has been demonstrated that GPR65 was associated with the increased level of cAMP, which can activate AMPK pathway. Interestingly, AMPK pathway may inhibit the activation of the JNK pathway, which is one of the most important pathways involved in osteoclastogenesis. We first detected the protein levels of the JNK pathway and AMPK pathway in CT-26 CM-treated early OCPs compared with normal early OCPs. As expected, the activated JNK protein was upregulated, and the level of activated AMPK $\alpha$ protein was significantly downregulated in early OCPs stimulated by CT-26 CM (Figures 6A-C). To test whether GPR65 regulates AMPK and JNK pathways in OCPs, plasmid containing GPR65 was transfected into OCPs. Interestingly, the activation of AMPK $\alpha$ was significantly enhanced after overexpression of GPR65, and the activation of JNK was prevented, and when cotransfected with AMPK $\alpha$ siRNA, the activation of JNK recovered in early OCPs treated with CT-26 CM (Figures 6D-F). These results showed that GPR65 inhibited activation of JNK through activating phosphorylation of AMPK $\alpha$ in OCPs in CRC microenvironment. To assess whether GPR65 can mediate activation of AMPK $\alpha$-regulated osteoclastogenic induction in CRC microenvironment, OCPs were induced into osteoclastogenic fate by stimulating with RANKL and M-CSF in the presence of CT-26 CM; meanwhile, plasmid containing GPR65 was transfected or cotransfected with AMPK $\alpha$ siRNA. TRAP staining showed the number of OCs was decreased in GPR65 overexpression group comparing with control group while the number increased after co-transfected with AMPK $\alpha$ siRNA (Figures 6G,H). Consistently, TRAP activity assay revealed overexpression of GPR65 significantly decreased the TRAP activity, but it can be rescued when co-transfected with AMPK $\alpha$ siRNA (Figure 6I). These results demonstrated that 


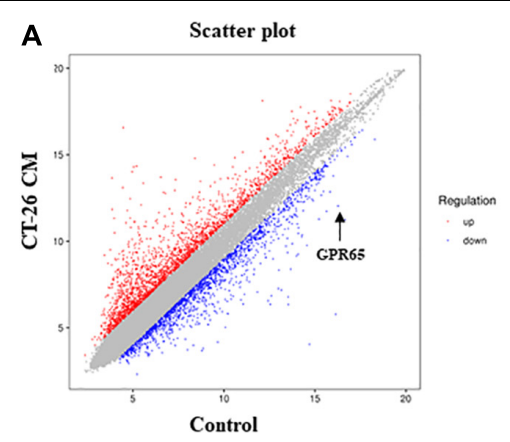

B
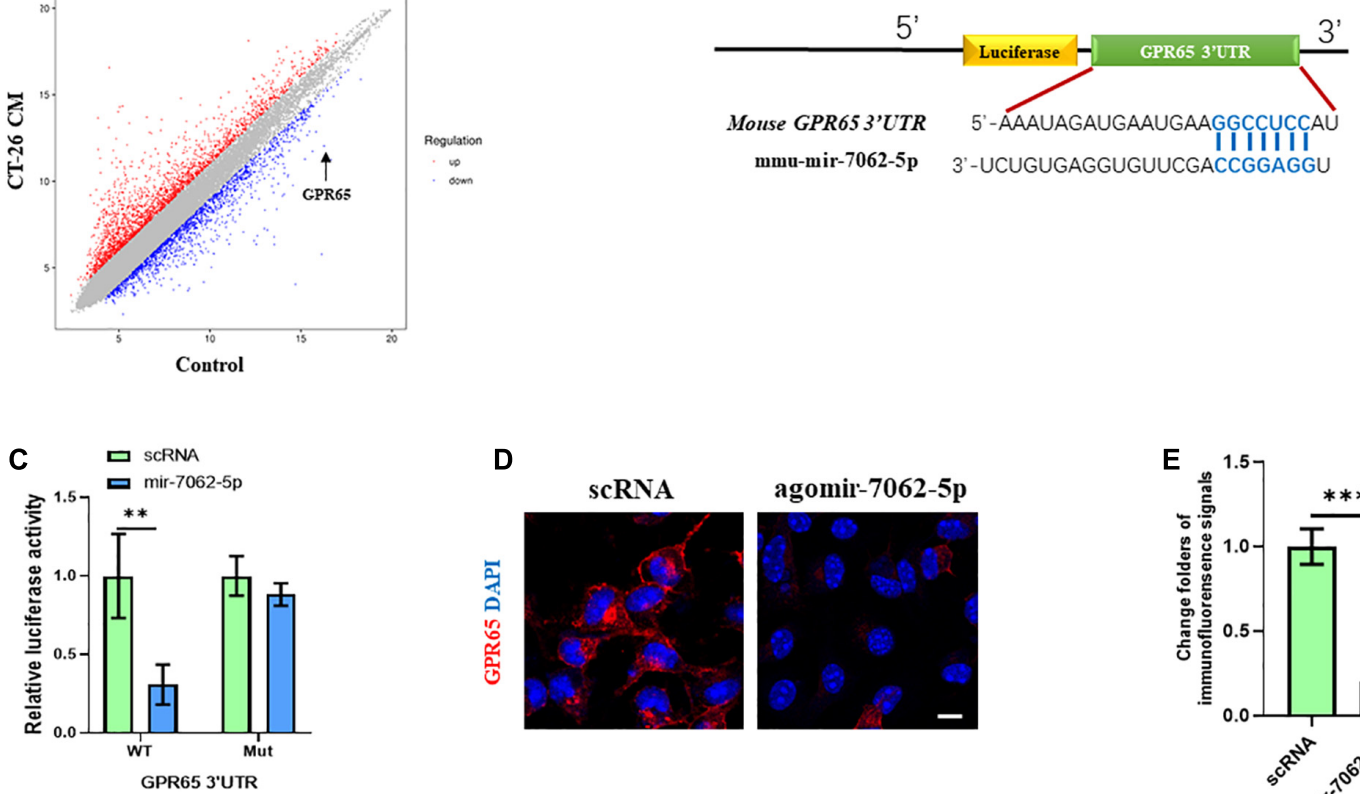

FIGURE 3 | GPR65 is the target gene of miRNA-7062-5p. (A) Scatter plots for microarray assay demonstrated differentially expressed mRNAs in early OCPs treated with CT-26 CM or DMEM. (B) The complementary sequences of miRNA-7062-5p were discovered in 3' UTR of GPR65 mRNA using TargetScan. (C) Dual-luciferase analysis showed miRNA-70652-5p inversely regulated the luciferase activity of plasmids containing wild-type 3' UTR of GPR65. (D) Immunofluorescence analysis revealed the protein expression of GPR65 in early OPCs after overexpression of miRNA-7062-5p (scale bar = $50 \mu \mathrm{m}$ ). (E) Quantification of immunofluorescence intensity in (D). ${ }^{* *} p<0.01,{ }^{* * *} p<0.001$.

A

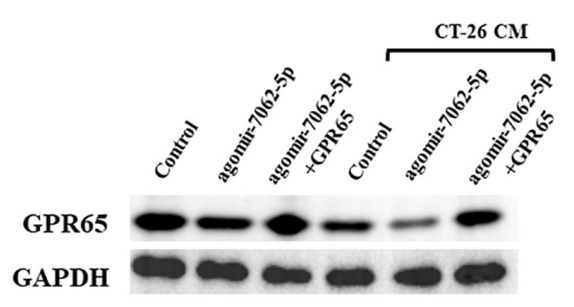

C

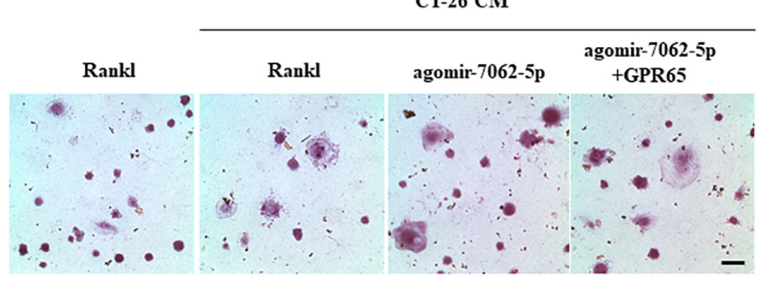

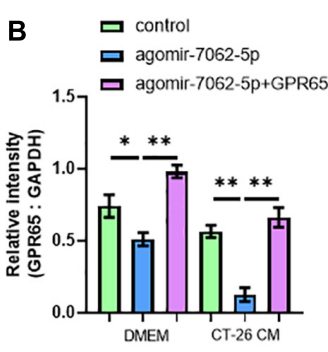

D

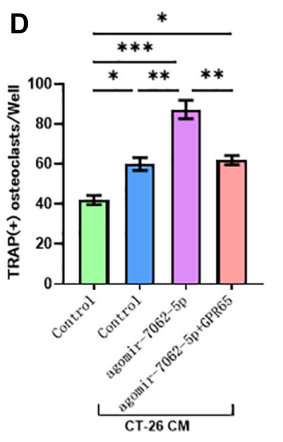

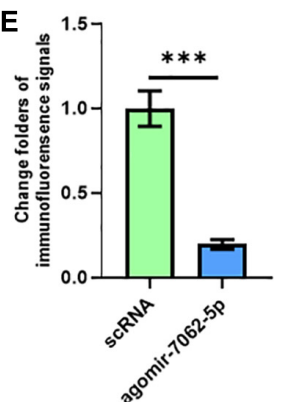


A

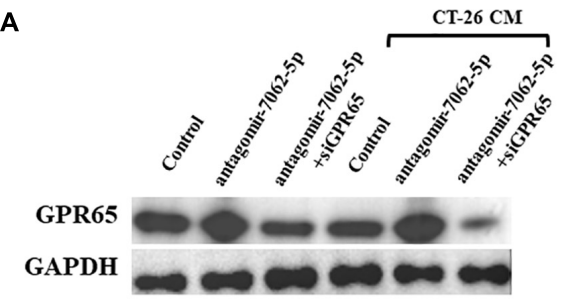

C

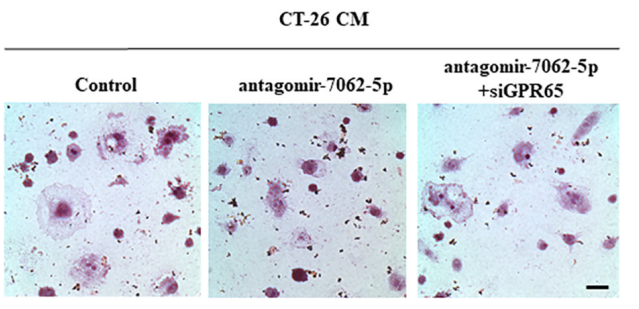

B

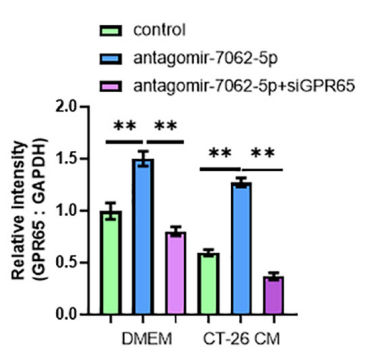

D

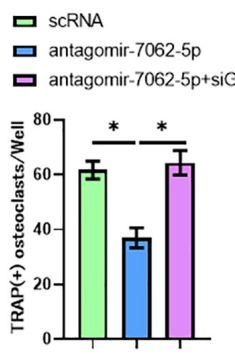

E

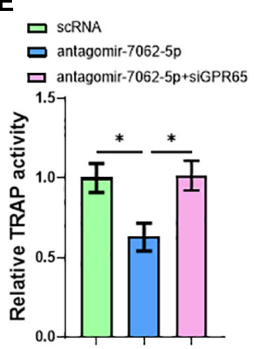

FIGURE 5 | Downregulation of GPR65 reverses the effect caused by inhibiting miRNA-7062-5p. (A) Western blot analysis demonstrated the protein level of GPR65 in early OCPs after transfection with antagomir-7062-5p alone or combination with GPR65 siRNA in the presence of CT-26 CM or in normal culture.

(B) Quantification of relative intensity in (A). (C) Representative images of TRAP staining demonstrating osteoclast formation of early OCPs induced by RANKL and M-CSF after transfection with antagomir-7062-5p alone or combination with GPR65 siRNA in the presence of CT-26 CM (scale bar $=250 \mu \mathrm{m}$ ). (D) Quantification of the number of osteoclasts per well in (C). (E) Quantification of relative TRAP activity per well in (C). ${ }^{*} p<0.05,{ }^{* *} p<0.01$.

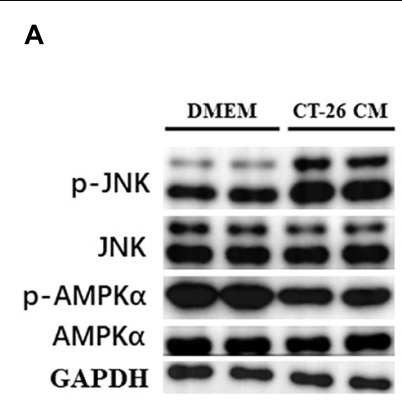

B

C
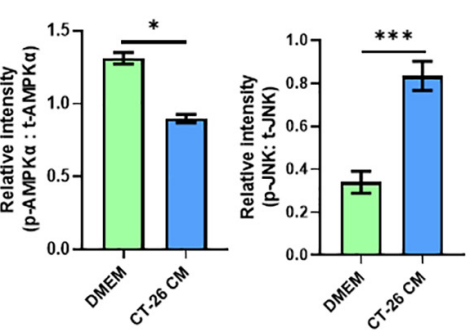

$\mathbf{G}$

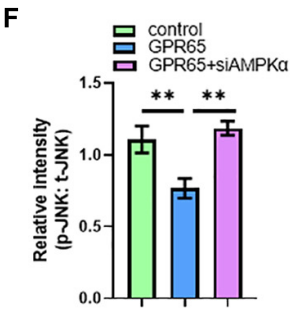

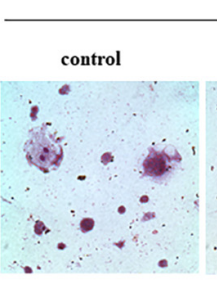

CT-26 CM

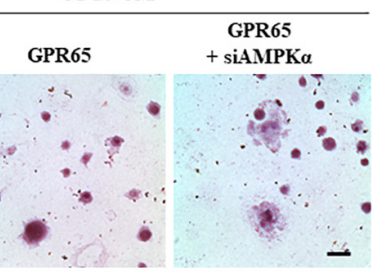

D

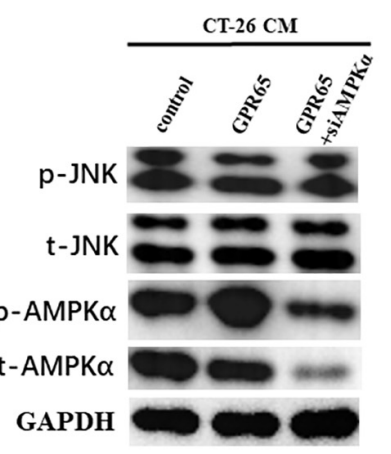

E

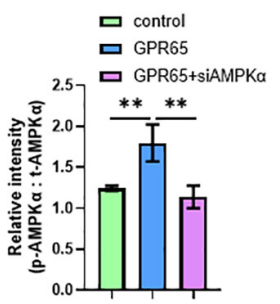

FIGURE 6 | Overexpression of GPR65 stimulates activation of AMPK pathway. (A) Western blot analysis showed protein level of activated AMPK and activated JNK compared with their own total proteins in early OCPs cultured in DMEM or CT-26 CM, respectively. (B) Quantification of relative intensity of phosphorylation of AMPKa compared with total AMPKain (A). (C) Quantification of relative intensity of phosphorylation of JNK compared with total JNK in (A). (D) Western blot analysis showed protein level of activated AMPK and activated JNK compared with their own total proteins in early OCPs after transfection with plasmid containing GPR65 with/without AMPK $\alpha$ siRNA in the presence of CT-26 CM. (E) Quantification of relative intensity of phosphorylation of AMPK $\alpha$ compared with total AMPKain (D). (F) Quantification of relative intensity of phosphorylation of JNK compared with total JNK in (D). (G) Representative images of TRAP staining demonstrating osteoclast formation of early OCPs induced by RANKL and M-CSF after transfection with plasmids containing GPR65 alone or combination with AMPK $\alpha$ siRNA in the presence of CT-26 CM (scale bar $=250 \mu \mathrm{m})$. (H) Quantification of relative TRAP activity per well in (G). (I) Quantification of the number of osteoclasts per well in (G). ${ }^{*} p<0.05,{ }^{* *} p<0.01,{ }^{* *} p<0.001$ 

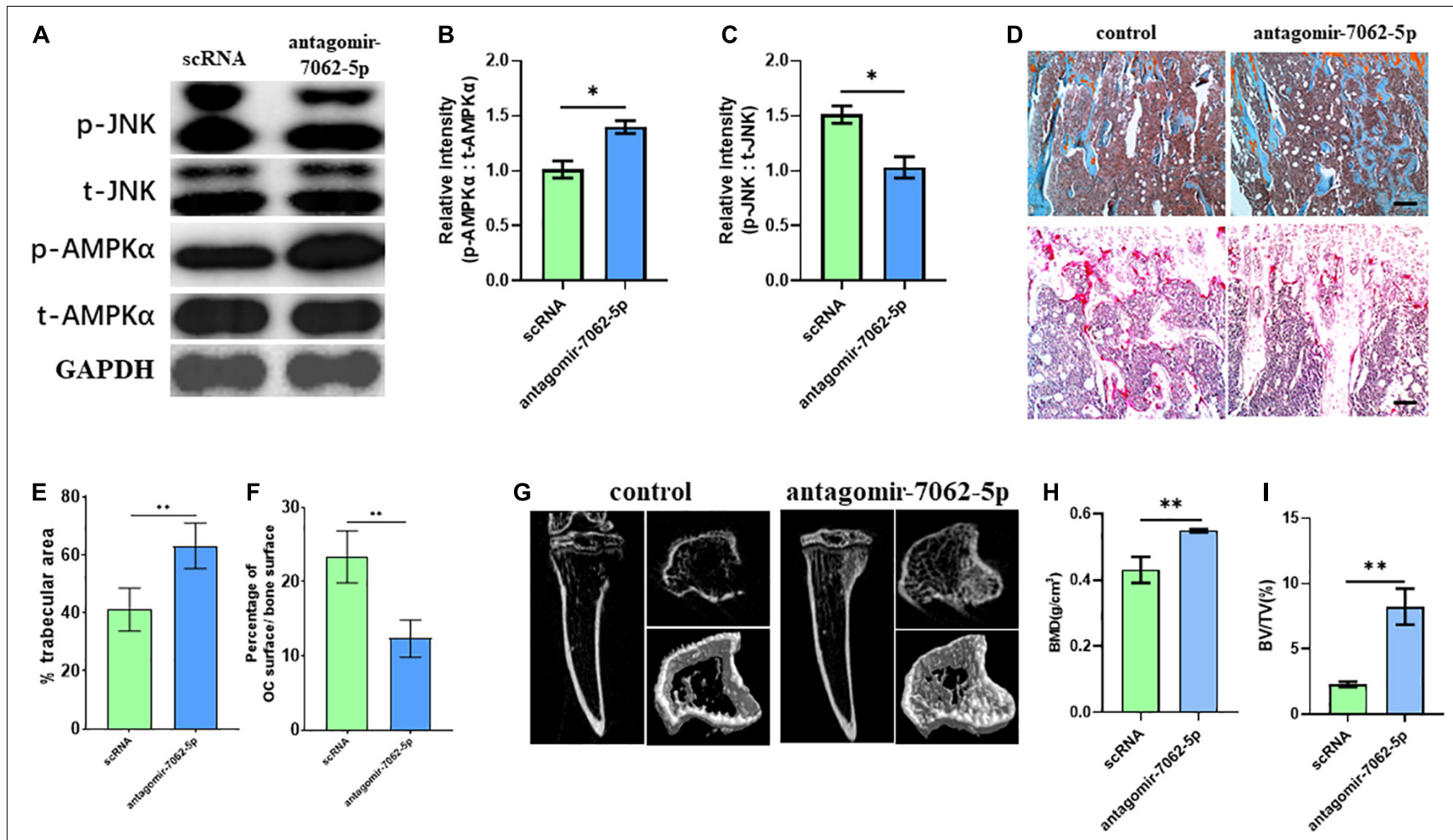

FIGURE 7 | Inhibition of miRNA-7062-5p attenuates bone resorption in bone metastatic model of CT-26 cell line in vivo. (A) Western blot analysis showed protein level of activated AMPK and JNK compared with its total protein in early OCPs sorted from antagomir-7062-5p-treated bone marrows after injection of CT-26 cells. (B) Quantification of relative intensity of phosphorylation of AMPK $\alpha$ compared with total AMPKain (A). (C) Quantification of relative intensity of phosphorylation of JNK compared with total JNK in (A). (D) Upper: representative images of safranin O staining in vivo after injection with antagomir-7062-5p in bone metastatic model. Down: representative images of TRAP staining in vivo after injection with antagomir-7062-5p in bone metastatic model (scale bar = $100 \mu \mathrm{m}$ ). (E) Quantification of percentage of trabecular area in upper images in (D). (F) Quantification of percentage of TRAP-positive osteoclast surface in total trabecular surface in down images in (D). (G) Micro-computed tomography examined the bone destruction after injection with antagomir-7062-5p in bone metastatic model. (H) Quantification of bone mineral density in tibias after injection with antagomir-7062-5p in bone metastatic model. (I) Quantification of BV/TV in tibias after injection with antagomir-7062-5p in bone metastatic model. ${ }^{*} p<0.05,{ }^{* *} p<0.01$.

GPR65-AMPK pathway regulated the osteoclastogenesis of OCPs in CRC microenvironment.

\section{Inhibition of miRNA-7062-5p Attenuates Bone Resorption Through Regulating GPR65/AMPK Pathway}

To detect whether anti-miRNA-7062-5p can inhibit osteoclastogenesis in vivo, antagomir-7062-5p was administered into tibias after injection of CT-26 cells, and the early OCPs were sorted out. Western blot revealed the activated JNK was inhibited, and the phosphorylated AMPK $\alpha$ was upregulated after treatment with antagomir-7062-5p (Figures 7A-C). Then, we tested whether inhibition of miRNA-7062-5p can prevent bone loss in the bone metastatic model of CRC in vivo. Notably, injection of antagomir-7062-5p significantly reduced number of OCs in vivo and restored trabecular area (Figures 7D-F). In addition, micro-computed tomography showed that the trabecular area was highly preserved after treatment with antagomir-7062-5p and the bone mineral density and trabecular bone volume fraction (BV/TV) were both improved (Figures 7G-I). These results demonstrated that antagmir-7062-5p can efficiently prevent bone resorption in bone metastasis of CRC.

\section{DISCUSSION}

In osteolytic tumors, bone destruction was positively associated with poor outcome (Powles et al., 1976; Kamalakar et al., 2014; Ma et al., 2018; Jie et al., 2019). It is well known that aberrant activation of osteoclasts contributes to bone resorption after tumor metastasis (Wilson et al., 2008; Das et al., 2011). Targeting osteoclastogenesis can prevent the progression of osteolysis and improve prognosis (Byrne et al., 2019).

Accumulating evidence has revealed that a large number of miRNAs play critical roles in regulating osteoclastogenesis; however, miRNAs involved in bone metastasis were elusive. Ell et al. identified that miR-16 and miR-378 promoted osteolytic bone metastasis in breast cancer and bladder cancer. These two miRNAs may be potential therapeutic targets and biomarkers, indicating that miRNAs could be critical to bone metastasis and tumor progression. As we described, although the incidence of bone metastasis in CRC is relatively less identified, the prognosis 


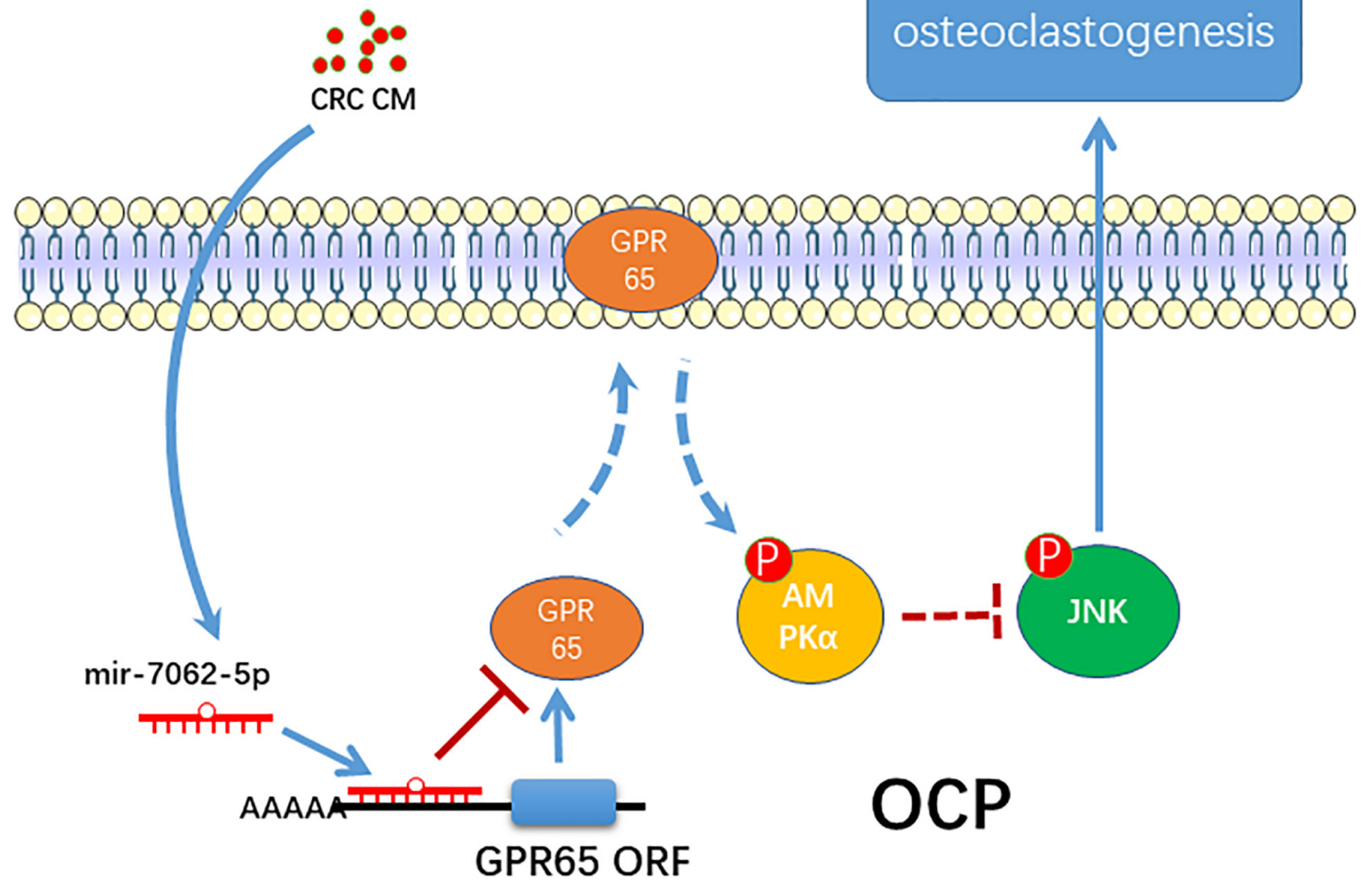

FIGURE 8 | The schematic of miR-7062-5p regulating osteoclastogenesis through targeting GPR65/AMPK pathway.

is worse. Numerous studies demonstrated that CRC is a kind of cancer that can be markedly regulated by miRNAs (Fang et al., 2012; Ma et al., 2013; Ye et al., 2013; Fu et al., 2014; Li et al., 2015; Xiao et al., 2015; Deng et al., 2016; Feiersinger et al., 2016; Noguchi et al., 2016; Fantini et al., 2017; Boriachek et al., 2018; Kandimalla et al., 2018; Lv et al., 2018). Typically, upregulated miRNAs in cancer promote the cancer, whereas downregulated miRNAs are likely to suppress tumor progression. MiR-155 and miR-503 were two reported miRNAs involved in invasion and metastasis of CRC (Noguchi et al., 2016; Liu et al., 2018). On the other hand, miR-320d, miR-203, miR-144, and miR-99b$5 \mathrm{p}$ prevented the proliferation, migration, or invasion in CRC cells (Xiao et al., 2015; Deng et al., 2016; Tang et al., 2019). Although correlation between miR-7062-5p and CRC was not identified in previous studies, we found that the expression of this miRNA can be upregulated in OCPs after stimulation by CRC cells and participate in osteoclast formation, indicating that a novel miRNA, miR-7062-5p, may specifically correlate with bone metastasis from CRC. Our findings together with previous studies revealed the diversity of miRNAs involved in different metastatic sites of CRC.

We here proved GPR65 is the target gene of miR-70625p. GPR65 is a member of G protein-coupled receptor family. Recently, the expression of GPR65 was found to be closely associated with intestinal diseases (Tcymbarevich et al., 2019a). Moreover, Tcymbarevich et al. revealed that deficiency in
GRP65 in macrophages positively promoted the progression of inflammatory bowel diseases (Tcymbarevich et al., 2019b). As OCPs derive from monocyte/macrophage cell lines, these findings implied that GRP65 may play an important role in regulating physiological process in macrophages. In this study, we identified that overexpression of GPR65 can reverse the osteoclastogenesis induced by miR-7062-5p, indicating that GPR65 plays a role in anti-osteoclast differentiation in bone metastasis of CRC. One study ever reported that TDAG8, another name of GPR65, inhibited osteoclastic activity in osteoporosis (Hikiji et al., 2014), which supports our findings.

Several pathways were reported to be downstream of GPR65, including AP-1 and MEK/ERK (Ryder et al., 2012; Xu et al., 2013), notably, considering close association between GPR65 and MEK/ERK and cAMP, which is linked to the AMPK pathway. In this study, we tested the change of activation of AMPK $\alpha$ and indicated that the overexpression of GPR65 activated phosphorylation of AMPK $\alpha$. Moreover, miR-7062-5p also downregulated activation of $\mathrm{AMPK} \alpha$, which can be rescued by overexpression of GPR65; thus, we demonstrated that AMPK is downstream of GPR65, and both of them can be regulated by miR-7062-5p. Numerous evidence demonstrated that activation of AMPK negatively regulated osteoclastogenesis (Dong et al., 2018; Tong et al., 2018; Mao et al., 2019). The AMPK pathway can inhibit the activation of the JNK pathway (Lin et al., 2016; Chen S. et al., 2018, Chen X. et al., 2018), which is a key way of 
stimulating osteoclast formation. In our study, overexpression of GPR65 can inhibit phosphorylation of JNK through activation of AMPK $\alpha$. Moreover, although overexpression of GPR65 inhibited osteoclast formation, this effect can be rescued by stimulating activation of $\mathrm{JNK}$, indicating that the negative effect of GPR65 on osteoclastogenesis was regulated by activation of JNK.

MiRNAs have been reported to be a promising therapeutic target in tumors. Our results showed that inhibition of miRNA7062-5p attenuated bone resorption after bone metastasis of CRC, which could be potentially used in the treatment of bone metastasis from CRC.

\section{CONCLUSION}

In summary, the present study proposes a novel role of miRNA7062-5p in osteoclast formation in bone metastasis of CRC through targeting GPR65, as well as regulating its downstream, AMPK pathway (Figure 8).

\section{DATA AVAILABILITY STATEMENT}

The data presented in the study are deposited in the GEO repository, accession number GSE182097. The link is: https:// www.ncbi.nlm.nih.gov/geo/query/acc.cgi?acc=GSE182097.

\section{REFERENCES}

Boriachek, K., Umer, M., Islam, M. N., Gopalan, V., Lam, A. K., Nguyen, N. T., et al. (2018). An amplification-free electrochemical detection of exosomal miRNA-21 in serum samples. Analyst 143, 1662-1669. doi: 10.1039/c7an01 $843 \mathrm{f}$

Byrne, N. M., Summers, M. A., and McDonald, M. M. (2019). Tumor cell dormancy and reactivation in bone: skeletal biology and therapeutic opportunities. JBMR Plus 3:e10125. doi: 10.1002/jbm4.10125

Chen, S., Zhao, L., Sherchan, P., Ding, Y., Yu, J., Nowrangi, D., et al. (2018). Activation of melanocortin receptor 4 with RO27-3225 attenuates neuroinflammation through AMPK/JNK/p38 MAPK pathway after intracerebral hemorrhage in mice. J. Neuroinflammation 15:106. doi: 10.1186/s12974-018-1140-6

Chen, X., Li, X., Zhang, W., He, J., Xu, B., Lei, B., et al. (2018). Activation of AMPK inhibits inflammatory response during hypoxia and reoxygenation through modulating JNK-mediated NF-kappaB pathway. Metabolism 83, 256-270. doi: 10.1016/j.metabol.2018.03.004

Clohisy, D. R., Palkert, D., Ramnaraine, M. L., Pekurovsky, I., and Oursler, M. J. (1996). Human breast cancer induces osteoclast activation and increases the number of osteoclasts at sites of tumor osteolysis. J. Orthop. Res. 14, 396-402. doi: 10.1002/jor.1100140309

Das, S., Samant, R. S., and Shevde, L. A. (2011). Hedgehog signaling induced by breast cancer cells promotes osteoclastogenesis and osteolysis. J. Biol. Chem. 286, 9612-9622. doi: 10.1074/jbc.M110.174920

Deng, B., Wang, B., Fang, J., Zhu, X., Cao, Z., Lin, Q., et al. (2016). MiRNA-203 suppresses cell proliferation, migration and invasion in colorectal cancer via targeting of EIF5A2. Sci. Rep. 6:28301. doi: 10.1038/srep2 8301

Dong, W., Qi, M., Wang, Y., Feng, X., and Liu, H. (2018). Zoledronate and high glucose levels influence osteoclast differentiation and bone absorption via the AMPK pathway. Biochem. Biophys. Res. Commun. 505, 1195-1202. doi: 10. 1016/j.bbrc.2018.10.059

\section{ETHICS STATEMENT}

The animal study was reviewed and approved by the Institutional Animal Care and Use Committee of Daping Hospital at Army Medical University.

\section{AUTHOR CONTRIBUTIONS}

LC carried out the experiments, analyzed and interpreted the data, and wrote the manuscript. YW analyzed part of data and prepared the manuscript. YW and XL carried out animal experiments and collected samples. XL interpreted part of data and collected samples. LZ and ZW conceived the study, designed the experiments, and wrote the manuscript. All authors read and approved the final manuscript.

\section{FUNDING}

The research reported was supported by the Daping Hospital (2019CXLCC011).

\section{ACKNOWLEDGMENTS}

We would like to thank all members of our laboratory for their work.

Fang, Y., Xiang, J., Chen, Z., Gu, X., Li, Z., Tang, F., et al. (2012). miRNA expression profile of colon cancer stem cells compared to non-stem cells using the SW1116 cell line. Oncol. Rep. 28, 2115-2124. doi: 10.3892/or.2012.2054

Fantini, S., Salsi, V., Reggiani, L., Maiorana, A., and Zappavigna, V. (2017). The miR-196b miRNA inhibits the GATA6 intestinal transcription factor and is upregulated in colon cancer patients. Oncotarget 8, 4747-4759. doi: 10.18632/ oncotarget. 13580

Feiersinger, F., Nolte, E., Wach, S., Rau, T. T., Vassos, N., Geppert, C., et al. (2016). MiRNA-21 expression decreases from primary tumors to liver metastases in colorectal carcinoma. PLoS One 11:e0148580. doi: 10.1371/journal.pone. 0148580

Fu, X., Meng, Z., Liang, W., Tian, Y., Wang, X., Han, W., et al. (2014). miR26a enhances miRNA biogenesis by targeting Lin28B and Zcchc11 to suppress tumor growth and metastasis. Oncogene 33, 4296-4306. doi: 10.1038/onc.2013. 385

Guo, J., Zeng, X., Miao, J., Liu, C., Wei, F., Liu, D., et al. (2019). MiRNA-218 regulates osteoclast differentiation and inflammation response in periodontitis rats through Mmp9. Cell Microbiol. 21:e12979. doi: 10.1111/cmi.12979

Guo, K., Zhang, D., Wu, H., Zhu, Q., Yang, C., and Zhu, J. (2018). MiRNA-199a-5p positively regulated RANKL-induced osteoclast differentiation by target $\mathrm{Mafb}$ protein. J. Cell. Biochem. 120, 7024-7031. doi: 10.1002/jcb.27968

Hikiji, H., Endo, D., Horie, K., Harayama, T., Akahoshi, N., Igarashi, H., et al. (2014). TDAG8 activation inhibits osteoclastic bone resorption. FASEB J. 28, 871-879. doi: 10.1096/fj.13-233106

Jie, Z., Xie, Z., Xu, W., Zhao, X., Jin, G., Sun, X., et al. (2019). SREBP-2 aggravates breast cancer associated osteolysis by promoting osteoclastogenesis and breast cancer metastasis. Biochim. Biophys. Acta Mol. Basis Dis. 1865, 115-125. doi: 10.1016/j.bbadis.2018.10.026

Kamalakar, A., Bendre, M. S., Washam, C. L., Fowler, T. W., Carver, A., Dilley, J. D., et al. (2014). Circulating interleukin-8 levels explain breast cancer osteolysis in mice and humans. Bone 61, 176-185. doi: 10.1016/j.bone.2014.01.015

Kandimalla, R., Gao, F., Matsuyama, T., Ishikawa, T., Uetake, H., Takahashi, N., et al. (2018). Genome-wide Discovery and Identification of a Novel miRNA 
Signature for Recurrence Prediction in Stage II and III Colorectal Cancer. Clin. Cancer Res. 24, 3867-3877. doi: 10.1158/1078-0432.CCR-17-3236

Kawamura, H., Yamaguchi, T., Yano, Y., Hozumi, T., Takaki, Y., Matsumoto, H., et al. (2018). Characteristics and prognostic factors of bone metastasis in patients with colorectal cancer. Dis. Colon Rectum. 61, 673-678. doi: 10.1097/ DCR.0000000000001071

Li, K., Chen, S., Cai, P., Chen, K., Li, L., Yang, X., et al. (2020a). MiRNA-483$5 p$ is involved in the pathogenesis of osteoporosis by promoting osteoclast differentiation. Mol. Cell Probes. 49:101479. doi: 10.1016/j.mcp.2019.10 1479

Li, S. S., Zhu, H. J., Li, J. Y., Tian, L. M., and Lv, D. M. (2020b). MiRNA-875-3p alleviates the progression of colorectal cancer via negatively regulating PLK1 level. Eur. Rev. Med. Pharmacol. Sci. 24, 1126-1133. doi: 10.26355/eurrev_ $202002 \_20163$

Li, W. C., Wu, Y. Q., Gao, B., Wang, C. Y., and Zhang, J. J. (2019a). MiRNA-574$3 p$ inhibits cell progression by directly targeting CCND2 in colorectal cancer. Biosci. Rep. 39:BSR20190976. doi: 10.1042/BSR20190976

Li, W., Chang, J., Wang, S., Liu, X., Peng, J., Huang, D., et al. (2015). miRNA-99b$5 p$ suppresses liver metastasis of colorectal cancer by down-regulating mTOR. Oncotarget 6, 24448-24462. doi: 10.18632/oncotarget.4423

Li, X., Luo, W., Hu, J., Chen, Y., Yu, T., Yang, J., et al. (2019b). Interleukin27 prevents LPS-induced inflammatory osteolysis by inhibiting osteoclast formation and function. Am. J. Transl. Res. 11, 1154-1169.

Li, Z., Zhang, W., and Huang, Y. (2018). MiRNA-133a is involved in the regulation of postmenopausal osteoporosis through promoting osteoclast differentiation. Acta Biochim. Biophys. Sin. (Shanghai) 50, 273-280. doi: 10.1093/abbs/gmy 006

Liang, M., Ma, Q., Ding, N., Luo, F., Bai, Y., Kang, F., et al. (2019). IL-11 is essential in promoting osteolysis in breast cancer bone metastasis via RANKLindependent activation of osteoclastogenesis. Cell Death Dis. 10:353. doi: 10. 1038/s41419-019-1594-1

Lin, K. C., Huang, D. Y., Huang, D. W., Tzeng, S. J., and Lin, W. W. (2016). Inhibition of AMPK through Lyn-Syk-Akt enhances FcepsilonRI signal pathways for allergic response. J. Mol. Med. (Berl) 94, 183-194. doi: 10.1007/ s00109-015-1339-2

Liu, N., Jiang, F., Han, X. Y., Li, M., Chen, W. J., Liu, Q. C., et al. (2018). MiRNA-155 promotes the invasion of colorectal cancer SW-480 cells through regulating the Wnt/beta-catenin. Eur. Rev. Med. Pharmacol. Sci. 22, 101-109. doi: 10.26355/eurrev_201801_14106

Luo, H., Sun, S., Li, P., Bu, D., Cao, H., and Zhao, Y. (2013). Comprehensive characterization of 10,571 mouse large intergenic noncoding RNAs from whole transcriptome sequencing. PLoS One 8:e70835. doi: 10.1371/journal. pone. 0070835

Lv, Q. L., Zhu, H. T., Li, H. M., Cheng, X. H., Zhou, H. H., and Chen, S. H. (2018). Down-regulation of miRNA-320c promotes tumor growth and metastasis and predicts poor prognosis in human glioma. Brain Res. Bull. 139, 125-132. doi: 10.1016/j.brainresbull.2018.02.009

Ma, G. T., Lee, S. K., Park, K. K., Park, J., Son, S. H., Jung, M., et al. (2018). Artemisinin-daumone hybrid inhibits cancer cell-mediated osteolysis by targeting cancer cells and osteoclasts. Cell Physiol. Biochem. 49, 1460-1475. doi: $10.1159 / 000493449$

Ma, Y., Li, W., and Wang, H. (2013). Roles of miRNA in the initiation and development of colorectal carcinoma. Curr. Pharm. Des. 19, 1253-1261. doi: $10.2174 / 138161213804805784$

Mao, Z., Zhu, Y., Hao, W., Chu, C., and Su, H. (2019). MicroRNA-155 inhibition up-regulates LEPR to inhibit osteoclast activation and bone resorption via activation of AMPK in alendronate-treated osteoporotic mice. IUBMB Life 71, 1916-1928. doi: 10.1002/iub.2131

Noguchi, T., Toiyama, Y., Kitajima, T., Imaoka, H., Hiro, J., Saigusa, S., et al. (2016). miRNA-503 promotes tumor progression and is associated with early recurrence and poor prognosis in human colorectal cancer. Oncology 90, 221-231. doi: 10.1159/000444493

Park, Y. R., Seo, S. Y., Kim, S. L., Zhu, S. M., Chun, S., Oh, J. M., et al. (2018). MiRNA-206 suppresses PGE2-induced colorectal cancer cell proliferation, migration, and invasion by targetting TM4SF1. Biosci. Rep. 38:BSR20180664. doi: $10.1042 /$ BSR20180664
Powles, T. J., Dowsett, M., Easty, G. C., Easty, D. M., and Neville, A. M. (1976). Breast-cancer osteolysis, bone metastases, and anti-osteolytic effect of aspirin. Lancet 1, 608-610. doi: 10.1016/s0140-6736(76) 90416-5

Ryder, C., McColl, K., Zhong, F., and Distelhorst, C. W. (2012). Acidosis promotes Bcl-2 family-mediated evasion of apoptosis: involvement of acid-sensing G protein-coupled receptor Gpr65 signaling to Mek/Erk. J. Biol. Chem. 287, 27863-27875. doi: 10.1074/jbc.M112.384685

Sun, S., Hang, T., Zhang, B., Zhu, L., Wu, Y., Lv, X., et al. (2019). miRNA-708 functions as a tumor suppressor in colorectal cancer by targeting ZEB1 through Akt/mTOR signaling pathway. Am. J. Transl. Res. 11, 5338-5356.

Tang, Y., Zhao, Y., Song, X., Song, X., Niu, L., and Xie, L. (2019). Tumor-derived exosomal miRNA-320d as a biomarker for metastatic colorectal cancer. J. Clin. Lab. Anal. 33:e23004. doi: 10.1002/jcla.23004

Tcymbarevich, I. V., Eloranta, J. J., Rossel, J. B., Obialo, N., Spalinger, M., Cosin-Roger, J., et al. (2019a). The impact of the rs8005161 polymorphism on G protein-coupled receptor GPR65 (TDAG8) pH-associated activation in intestinal inflammation. BMC Gastroenterol 19:2. doi: 10.1186/s12876-0180922-8

Tcymbarevich, I., Richards, S. M., Russo, G., Kühn-Georgijevic, J., Cosin-Roger, J., Baebler, K., et al. (2019b). Lack of the pH-sensing Receptor TDAG8 [GPR65] in Macrophages Plays a Detrimental Role in Murine Models of Inflammatory Bowel Disease. J. Crohns. Colitis. 13, 245-258. doi: 10.1093/ecco-jcc/ jjy152

Tong, X., Gu, J., Song, R., Wang, D., Sun, Z., Sui, C., et al. (2018). Osteoprotegerin inhibit osteoclast differentiation and bone resorption by enhancing autophagy via $\mathrm{AMPK} / \mathrm{mTOR} / \mathrm{p} 70 \mathrm{~S} 6 \mathrm{~K}$ signaling pathway in vitro. J. Cell Biochem. 120, 1630-1642. doi: $10.1002 /$ jcb. 27468

Wilson, T. J., Nannuru, K. C., Futakuchi, M., Sadanandam, A., and Singh, R. K. (2008). Cathepsin G enhances mammary tumor-induced osteolysis by generating soluble receptor activator of nuclear factor-kappaB ligand. Cancer Res. 68, 5803-5811. doi: 10.1158/0008-5472.CAN-07-5889

Xiao, R., Li, C., and Chai, B. (2015). miRNA-144 suppresses proliferation and migration of colorectal cancer cells through GSPT1. Biomed. Pharmacother. 74, 138-144. doi: 10.1016/j.biopha.2015.08.006

Xu, H., Chen, X., Huang, J., Deng, W., Zhong, Q., Yue, C., et al. (2013). Identification of GPR65, a novel regulator of matrix metalloproteinases using high through-put screening. Biochem. Biophys. Res. Commun. 436, 96-103. doi: $10.1016 /$ j.bbrc.2013.05.065

Ye, J., Wu, X., Wu, D., Wu, P., Ni, C., Zhang, Z., et al. (2013). miRNA-27b targets vascular endothelial growth factor $\mathrm{C}$ to inhibit tumor progression and angiogenesis in colorectal cancer. PLoS One 8:e60687. doi: 10.1371/journal. pone. 0060687

Zhang, Y., Ma, L. N., and Xie, Y. (2020). MiRNA-802 inhibits the metastasis of colorectal cancer by targeting FOXE1. Eur. Rev. Med. Pharmacol. Sci. 24, 1778-1785. doi: 10.26355/eurrev_202002_20355

Zhao, H., Zhang, J., Shao, H., Liu, J., Jin, M., Chen, J., et al. (2017). miRNA340 inhibits osteoclast differentiation via repression of MITF. Biosci. Rep. 37:BSR20170302. doi: 10.1042/BSR20170302

Conflict of Interest: The authors declare that the research was conducted in the absence of any commercial or financial relationships that could be construed as a potential conflict of interest.

Publisher's Note: All claims expressed in this article are solely those of the authors and do not necessarily represent those of their affiliated organizations, or those of the publisher, the editors and the reviewers. Any product that may be evaluated in this article, or claim that may be made by its manufacturer, is not guaranteed or endorsed by the publisher.

Copyright $\odot 2021$ Chen, Wang, Lu, Zhang and Wang. This is an open-access article distributed under the terms of the Creative Commons Attribution License (CC BY). The use, distribution or reproduction in other forums is permitted, provided the original author(s) and the copyright owner(s) are credited and that the original publication in this journal is cited, in accordance with accepted academic practice. No use, distribution or reproduction is permitted which does not comply with these terms. 